\title{
Transient infantile hypertriglyceridemia and hepatosteatosis
}

INSERM

\section{Source}

INSERM. (1999). Orphanet: an online rare disease and orphan drug data base. Transient infantile hypertriglyceridemia and hepatosteatosis. ORPHA:300293

Transient infantile hypertriglyceridemia and hepatosteatosis is a rare, genetic, hepatic disease characterized by massive hepatomegaly, moderate to severe, transient hypertriglyceridemia and hepatic steatosis (followed by fibrosis), manifesting in infancy with failure to thrive, vomiting, an enlarged abdomen and a fatty liver. Reduction or normalization of triglyceride serum levels occurs with advancing age. 